\title{
Prognostic assessment in patients with hepatic encephalopathy
}

\author{
Rita García-Martínez, Macarena Simón-Talero and Juan Córdoba* \\ Servei de Medicina Interna-Hepatologia. Hospital Vall d'Hebron, Departament de Medicina, Universitat \\ Autònoma de Barcelona, Centro de Investigación Biomédica en Red de Enfermedades Hepáticas y Digestivas \\ (CIBEREHD), Spain
}

\begin{abstract}
Hepatic encephalopathy (HE) is a common complication of liver failure that is associated with poor prognosis. However, the prognosis is not uniform and depends on the underlying liver disease. Acute liver failure is an uncommon cause of HE that carries bad prognosis but is potentially reversible. There are several prognostic systems that have been specifically developed for selecting patients for liver transplantation. In patients with cirrhosis the prognosis of the episode of HE is usually dictated by the underlying precipitating factor. Acute-on-chronic liver failure is the most severe form of decompensation of cirrhosis, the prognosis depends on the number of associated organ failures. Patients with cirrhosis that have experienced an episode of HE should be considered candidates for liver transplant. The selection depends on the underlying liver function assessed by the Model for End-stage Liver Disease (MELD) index. There is a subgroup that exhibits low MELD and recurrent HE, usually due to the coexistence of large portosystemic shunts. The recurrence of HE is more common in patients that develop progressive deterioration of liver function and hyponatremia. The bouts of HE may cause sequels that have been shown to persist after liver transplant.
\end{abstract}

Keywords: Hepatic encephalopathy, prognosis, acute liver failure, acute-on-chronic liver failure, liver transplantation

\section{Abbreviations}

$\begin{array}{ll}\text { HE } & \text { Hepatic Encephalopathy } \\ \text { MELD } & \text { Model for End-stage Liver Disease } \\ \text { ALF } & \text { Acute Liver Failure } \\ \text { HBV } & \text { Hepatitis B Virus } \\ \text { TIPS } & \text { Transjugular Intrahepatic Portosystemic Shunts } \\ \text { HCC } & \text { Hepatocellular Carcinoma } \\ \text { BCLC } & \text { Barcelona Clinic Liver Cancer } \\ \text { AOLF } & \text { Acute-on-chronic liver failure } \\ \text { MARS } & \text { Molecular Absorbent Recirculating System } \\ \text { EEG } & \text { Electroencephalogram } \\ \text { CFF } & \text { Critical Flicker Frequency } \\ \text { LT } & \text { Liver Transplantation }\end{array}$

\section{Introduction}

Hepatic encephalopathy (HE) is usually interpreted as a sign of liver failure and has ominous considera-

* Address for correspondence: Juan Córdoba, Servei de Medicina Interna-Hepatologia. Hospital Vall d'Hebron. Paseo Vall d'Hebron 119, Barcelona, 08035, Spain. E-mail: jcordoba@vhebron.net. tions. However, as for other complications of cirrhosis (e.g. jaundice, hepatocellular carcinoma, hepatorenal syndrome...), the prognosis of patients with HE is not uniform. Establishing the prognosis is difficult and requires a precise assessment of neurological and hepatic function [1]. HE is characterized by a myriad of neurological manifestations, diverse underlying liver disorders and a variety of precipitating factors [2]; all of them may affect prognosis. The approach to the patient with HE should be performed according to the underlying disorders, as is recognized in the classification (Table 1). This article reviews the assessment of prognosis in patients with HE, based on the underlying liver disease and the severity of neurological manifestations. Unfortunately, the majority of studies have not classified the patients according to the type of liver disease and the availability of data in some areas is limited.

\section{Acute liver failure}

The development of HE in a patient that was previously asymptomatic should prompt the diagnosis of 
Table 1

Classification of hepatic encephalopathy

\begin{tabular}{|c|c|c|c|}
\hline Liver disease & Types & Subtypes & Features \\
\hline A-Acute liver failure & & & $\begin{array}{l}\text { Neurological manifestations include typically the presence of intracranial hyperten- } \\
\text { sion. The liver was previously healthy, the patient has the possibility to completely } \\
\text { regenerate and recover the liver function }\end{array}$ \\
\hline B-Portosystemic by-pass & & & $\begin{array}{l}\text { Neurological manifestations secondary to large portosystemic shunts that may be } \\
\text { congenital or surgical induced. There is no underlying liver disease. }\end{array}$ \\
\hline \multirow[t]{5}{*}{ C-Cirrhosis } & EpisodicdI & Precipitated & $\begin{array}{l}\text { Secondary to gastrointestinal hemorrhage, constipation, excessive protein intake, } \\
\text { infection, renal failure, dehydration, electrolyte disturbance. }\end{array}$ \\
\hline & & Spontaneous & $\begin{array}{l}\text { Without recognized precipitating factors. Usually associated with large portosys- } \\
\text { temic shunts (spontaneous, surgical, TIPS) }\end{array}$ \\
\hline & Persistent & Mild & $\begin{array}{l}\text { Chronic cognitive or motor manifestations that impact negatively on social and } \\
\text { occupational activities but do not cause dependency }\end{array}$ \\
\hline & & Severe & $\begin{array}{l}\text { Chronic manifestations that cause dependency (dementia, paraplegia, parkinson- } \\
\text { ism...) }\end{array}$ \\
\hline & Minimal & & $\begin{array}{l}\text { Cognitive disturbances detected by neuropsychological or neurophysiological tests } \\
\text { that are not evident in the standard neurological examination }\end{array}$ \\
\hline
\end{tabular}

${ }^{*}$ The classification is based on the consensus of Viena (1).

ITTe term recurrent HE refers to patients that have experienced more than 2 episodes of HE within the last six months.

acute liver failure (ALF). This is a rare condition in which rapid deterioration of liver function results in altered mentation and coagulopathy [3]. The presence of $\mathrm{HE}$ is a requirement for the diagnosis; in other words, ALF cannot be diagnosed in the absence of HE. The most prominent causes of ALF are drug induced liver injury, viral hepatitis, autoimmune liver disease and shock [4]. However, approximately $20 \%$ of cases have no discernible cause [5].

ALF often affects young persons and carries a high morbidity and mortality. It is unclear why some patients with the same apparent degree of severity and the same aetiology of liver failure have different outcomes. Indeed after the occurrence of encephalopathy, ALF patients can die without liver transplantation while others will recover either in a few hours or in a few days. Prior to transplantation, most series suggested less than $15 \%$ survival; liver transplantation has made it possible to achieve survival greater than $65 \%$ [5]. The prognosis of ALF depends on many factors. The most important are age, aetiology of ALF and clinical and biological status on admission and at the peak of deterioration.

ALF can occur through distinct pathways according to aetiologies; there is probably more than one mechanism responsible for ALF. High level of spontaneous recovery without liver transplantation $(>50 \%)$ is seen in patients with paracetamol overdose, acute hepatitis A infection, liver shock, and pregnancy- related ALF. To the contrary, prognosis is poor in patients with ALF due to indeterminate causes, drug-intoxication other than paracetamol, hepatitis B virus (HBV) infection, autoimmune hepatitis, Wilson's disease, and Budd Chiari syndrome.
There are several prognostic systems that have been specifically developed for selecting ALF patients for liver transplantation [6]. Overall, such prognostic scores have proven to have acceptable specificity but have low sensitivity to determine outcome. Since the currently available prognostic scoring systems do not adequately predict outcome and determine candidacy for liver transplant the decision to proceed to liver transplant could not rely only upon them [3]. Each centre should establish its own transplant policy according to local epidemiology and experience and use the prognostic criteria as a tool, but not as definitive criteria. A flexible use of them with continuous monitoring seems to be the wiser approach. There is no unanimously accepted prognostic system. The most widely used are the King's College criteria [7] and the Clichy-Villejuif criteria [8] (Table 2). The King's College criteria have shown positive predictive values ranging from just below $70 \%$ to nearly $100 \%$ and negative predictive values ranging from $25 \%$ to $94 \%[9,10]$. The Clichy-Villejuif predicted death in acute viral hepatitis cases with a positive predictive value of $82 \%$ and a negative predictive value of 98 but subsequent studies have shown these criteria to be less accurate than King's College criteria in predicting outcome [11].

The neurological status influences survival; severe HE (grade 3-4) upon admission and during hospitalization is a significant determinant of poor outcome [5]. For this reason, some centres have decided to use the severity of $\mathrm{HE}$ as a main determinant to select patients for liver transplant [12]. Advanced $\mathrm{HE}$ is a marker of the severity of liver function and of the presence of intracranial hypertension, a common complication 
Table 2

Prognostic criteria for Patients With ALF

\begin{tabular}{lll}
\hline Clichy-Villejuif & Age & Criteria \\
& Under 30 years: & HE stage 3 or $4+$ factor $\mathrm{V}<20 \%$ \\
& Over 30 years: & HE stage 3 or $4+$ factor $\mathrm{V}<30 \%$ \\
King's College & Etiology & Criteria \\
& Paracetamol & Lactate $>3.5 \mathrm{Mmol} / \mathrm{L}$, \\
& - Arterial $\mathrm{pH}<7.3$, or lactate $>3 \mathrm{mmol} / \mathrm{L}$ after adequate volume \\
& resuscitation \\
& or \\
& - HE stage 3 or $4+$ creatinine $>300 \mathrm{mcm} / \mathrm{L}+$ INR $>6.5$ \\
& \\
& & INR $>6.7$ \\
& or any three of the following: \\
& - drug toxicity or indeterminate cause \\
& - age $<10$ or $>40$ years \\
& - jaundice to coma interval $>7$ days \\
& - bilirubin $>300$ mcm/L \\
& - INR $>3.5$ \\
&
\end{tabular}

of ALF that is responsible for an important number of deaths secondary to brain herniation. Recent studies have shown that the concentration of ammonia in plasma can be used to predict the development of intracranial hypertension, which may help to select patients who should undergo intracranial pressure monitoring. The risk of brain herniation is higher for plasma ammonia concentration above 200 microM/L [13].

\section{Acute $\mathrm{HE}$ in cirrhosis}

The importance of HE in determining the prognosis of patients with cirrhosis has been acknowledged in the most widely used system that assesses the severity of liver failure: the Child-Pugh scoring system. Howev$\mathrm{er}$, the experience with patients that have undergone portosystemic shunts or Transjugular Intrahepatic Portosystemic Shunts (TIPS) showed that the development of HE in these patients is independent of survival. Clinical experience also indicates that there is a subgroup of patients with cirrhosis that in spite of developing multiple episodes of HE have a long-life. In accordance to these experiences, the MELD score, which has replaced the Child-Pugh system in the selection of patients for liver transplant, does not include HE.

Patients with cirrhosis presenting with acute HE should undergo a diagnostic process that assesses the presence of precipitating factors and the severity of liver function (Fig. 1). It is possible to classify patients according to underlying liver disease and the previous performance status. For those with hepatocellular carcinoma (HCC), the Barcelona Clinic Liver Cancer (BCLC) system is the most appropriate [14]. In pa- tients with cirrhosis that have not developed HCC a classification in stages has been proposed according to the presence of ascites and varices [15]. These stages have been established by combining data from 2 large natural history studies, recently refined by the results of an analysis of the prognostic significance of infection in cirrhosis (Fig. 2) [16]. In this classification stage 1 is defined by the absence of ascites and oesophageal varices (mortality at 1 year: 1\%), stage 2 by the presence of oesophageal varices without bleeding (increase in mortality: $3 \%$ ), stage 3 by the presence of ascites with or without oesophageal varices (increase in mortality: 20\%) and stage 4 by the occurrence of variceal bleeding with or without ascites (increase in mortality to $57 \%$ ). Newly proposed stages 5 (development of infections) and 6 (hepatorenal syndrome) define stages of more advanced severity. Interestingly, $\mathrm{HE}$ is not present in any of these stages, indicating that while HE is frequent at the time of death, is not a reliable indicator of prognosis in cirrhosis. This may be explained by the role of portosystemic shunting in the pathogenesis of $\mathrm{HE}$ and by the presence of precipitating factors that may directly affect prognosis.

\subsection{Acute-on-chronic liver failure}

A major pathogenic element in the development of $\mathrm{HE}$ is the presence of portosystemic shunting, which may be intrahepatic or extrahepatic [1]. Severe liver diseases cause intrahepatic shunts due to endothelization of sinusoids and insufficient liver mass. They are recognized by the development of coagulopathy (longer prothrombin time) and jaundice (high bilirubin). The most characteristic extreme example of this situation 


\section{Cirrhosis + acute encephalopathy}
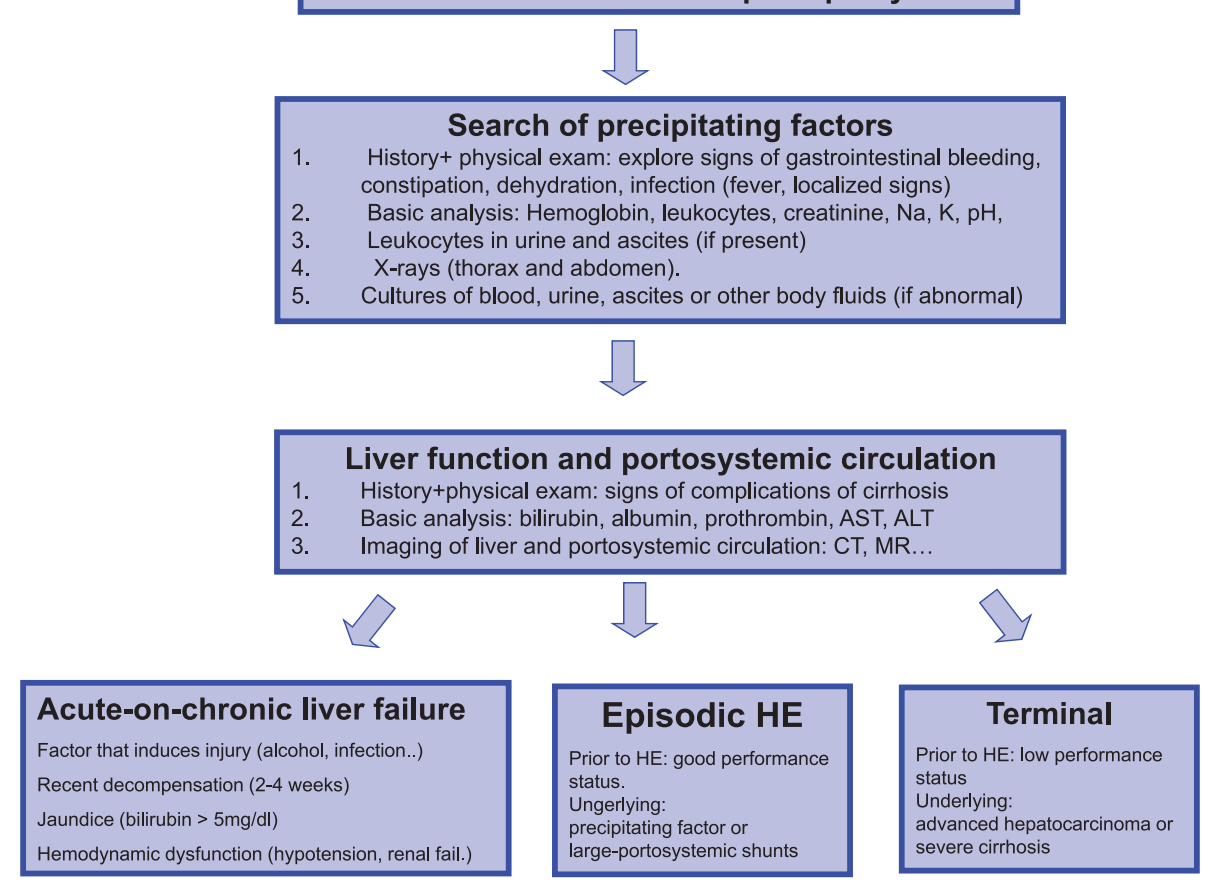

Fig. 1. The assessment of a patient with cirrhosis and an acute change in mental state should be initiated by investigating the existence of precipitanting factors. The assessment should be completed with blood tests and imaging studies that evaluate liver function and portosystemic circulation. According to the results the patients are classified as: a) episodic HE b) acute-on-chronic liver failure or c) terminal liver disease, and managed accordingly.

is acute-on-chronic liver failure (AOLF) and terminal irreversible cirrhosis secondary to advanced HCC. Patients that develop $\mathrm{HE}$ as the final event of liver failure are usually identified in this terminal situation before the occurrence of HE; they develop a progressive decline in their quality of life and show a poor performance status for weeks before the development of HE.

AOLF has been coined to refer to a situation that is poorly defined: severe liver failure that develops in a relatively short period of time (typically less than 4 weeks), secondarily to a precipitating event (e.g. acute alcoholic hepatitis), in a patient with previously compensated cirrhosis [17]. The severity of liver failure is recognized by high bilirubin, prolonged prothrombin time and the development of failure of other organs (kidney failure, hypotension, respiratory failure...).

Patients with AOLF are critically ill and are usually managed in a critical care environment. Due to the lack of diagnostic criteria, the prognosis of AOLF has not been specifically studied. In a population that probably corresponds to AOLF (cirrhotic patients with MELD $>18$ and signs of systemic inflammatory response) the in-hospital mortality is around $50 \%[18,19]$.
The most important factor that determines prognosis in AOLF is the development of multiorgan failure. Scoring systems that have been developed for critically ill patients (SOFA, APACHE II and III) have shown a better reliability than the Child-Pugh or the MELD to identify patients with bad prognosis. These systems provide operational criteria to define extra-hepatic organ failure. It has been shown that patients with 2 organ failures or undergoing the artificial support of 2 organic systems have a high mortality (approximately $75 \%$ ). This mortality approaches $100 \%$ for 3 or more failing organs [20].

One important determinant of prognosis for patients with AOLF and severe HE (grade 3-4) is the lack of improvement of HE during the first week of treatment. This was clearly shown in a clinical trial that investigated the effects of MARS (Molecular Absorbent Recirculating System) therapy for severe HE [21]. The study included 70 patients (MELD $30 \pm 10$ ) that were randomized to receive MARS (up to 5 days) or standard medical therapy alone. Treatment with MARS resulted in a more rapid improvement of HE and for those with MELD>30 in a better 2-week survival. The authors found that in this population, which had a predicted 


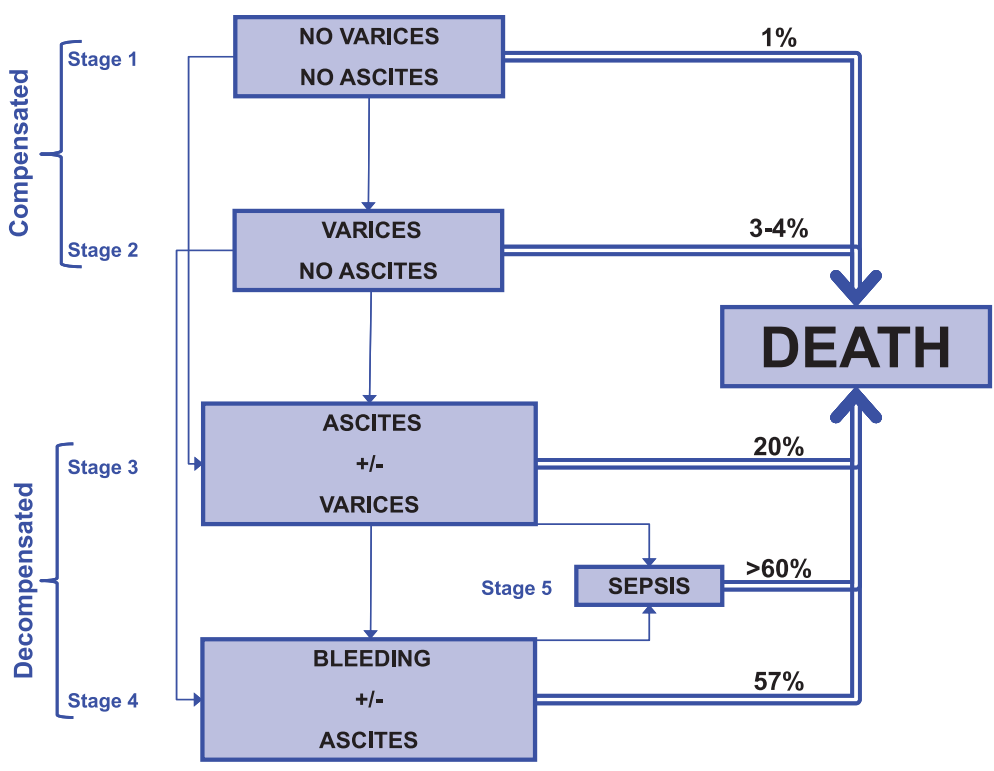

Fig. 2. Clinical outcome in cirrhosis and classification of patients in five different stages according to the presence of cirrhosis, varices and sepsis: stage 1: without varices or ascites, stage 2: with varices and without ascites, stage 3 : ascites \pm varices, and stage 4: bleeding \pm ascites. Mortality at 1-year increases in each stage. The development of sepsis is more likely in stages 3 and 4 and the risk of dying is higher. The development of sepsis has been proposed to as stage 5 (modified from Arvaniti V et al. [16]).

mortality above $75 \%$, the two factors that were predictors of a 4-week survival were performance of liver transplantation and improvement of HE by 2 grades in the 5 days study period.

In conclusion, patients with HE and AOLF have a bad prognosis and if possible liver transplant should be a priority. For those awaiting transplant, MARS could become part of a bridging strategy. Patients with AOLF that develop more than two organ failure or exhibit lack of improvement of severe HE after 5 days of treatment have a very poor prognosis. In this situation, supportive therapies may be considered futile and the therapeutic efforts could be limited.

\subsection{Episodic $H E$}

Episodic HE in cirrhotic patients is associated with short life expectancy. One study that investigated survival after the first episode of HE found a cumulative survival a one year of $42 \%$ and at 3 years of $23 \%$ [22]. These results were obtained in the 1990s in a liver unit with a large experience in management of cirrhosis. Since the data were obtained in a referral centre with a transplant program, they may be biased towards more severe patients. However, the data are in agreement with the results previously reported by other authors $[23,24]$. The authors proposed that the prog- nosis in cirrhotic patients developing HE has not substantially changed during the last decades and that all patients that have developed HE should be considered liver transplant candidates.

The outcome of HE in patients with cirrhosis that do not fulfil the criteria for AOLF is usually determined by the precipitating factor. This concept proceeds from the experience in clinical trials $[25,26]$. The precipitating factor is a clinical event that does not cause a direct injury to the liver or to the portal-systemic circulation but is responsible for the acute change in the mental state. Precipitating factors appear to act by increasing the generation of putative toxins or enhancing the effects of the toxins on the central nervous system. They are temporally related to the development of HE and their correction to the re-establishment of consciousness. Several factors are traditionally considered under this category (gastrointestinal bleeding, constipation, excessive protein intake, dehydration, electrolyte disturbances, renal failure and infection), and are thought to explain the majority of episodes of HE. However, a significant number of episodes are not related to a precipitating factor [27].

Patients that have survived to an episode of $\mathrm{HE}$ should be evaluated for liver transplant. The system to decide if a patient is an appropriate candidate is not different from the currently used in most centres. The 
prognosis after HE is clearly related to the severity of liver failure. In the study by Bustamante [22] the authors related survival to bilirubin, albumin, prothrombin time, urea and potassium. This supports the use of MELD in deciding which patients that have developed HE should undergone liver transplant. Nevertheless, one retrospective study shows that HE provides additional prognostic information than the one given by MELD [28]. Future studies should confirm this finding, before modifying the current transplant policy.

\subsection{Large portosystemic shunts}

The development of HE may be determined by large extrahepatic portosystemic shunts. The experience with surgical porto-caval anastomosis and with TIPS indicates that these are high-risk patients for HE. According to studies in patients with TIPS, the prognosis in this situation, as in patients without TIPS, is determined by parameters of liver function that are included in the MELD [29]. The development of severe $\mathrm{HE}$ (grade 3-4) is associated with a higher degree of mortality, probably because identifies hospitalized patients with a severe decompensation [28]. Interestingly, the prognosis is better in patients with TIPS than in those without TIPS, supporting the notion than in the presence of large portosystemic shunts the severity of HE is less important than in the presence of other precipitating factors.

There is a group of patients with cirrhosis and large spontaneous shunts (non-procedural shunts). The prognosis of these patients appears to be similar to the one of those that have undergone procedural shunts [30]. Patients with large spontaneous shunts are usually characterized by good parameters of liver function and do not fulfil the criteria for liver transplant. Large shunts may be suspected by a history of frequents episodes of $\mathrm{HE}$, lack of variceal haemorrhage and relatively preserved liver function (lack of coagulopathy and jaundice) [31]. These patients should undergo imaging of the portosystemic circulation. The survival of patients with large spontaneous portosystemic shunts has not been specifically investigated. The experience in patients with surgical shunts suggests that this condition has a much better outcome than the development of $\mathrm{HE}$ in the absence of large shunts.

\section{Recurrence of $\mathrm{HE}$}

HE is characterized by its elevated tendency to relapse; it has been estimated that approximately half of the patients that survive to an episode of HE will recur during the following year [32]. One of the factors that have been proposed to identify the subjects that will suffer a new episode of HE is the presence of minimal HE. However, part of the prognostic significance can be attributed to the presence of more severe liver failure among patients with minimal HE [33]. The combination Child-Pugh $\mathrm{B} / \mathrm{C}$ and minimal $\mathrm{HE}$, detected by electroencephalogram (EEG) [34] or by critical flicker frequency (CFF) [35] identifies those patients at a higher risk of recurrence.

In patients with minimal $\mathrm{HE}$ the presence of a high ammonia value after an oral glutamine challenge is associated with the development of HE [36]. This finding was explained by a higher activity of intestinal glutaminase, an enzyme that is present in the intestinal mucosa and deaminates glutamine. The activity of glutaminase may determine the hyperammonemia that follows the digestion of proteins. One interesting observation has been the identification of specific polymorphisms in the glutaminase gen that may increase the activity of intestinal glutaminase and through this mechanism predisposes to the occurrence of episodic HE in affected individuals [37]. If confirmed, genetic polymorphism should be included in the group of predisposing conditions. Apart from identifying high-risk patients, the assessment of the polymorphism may initiate the era of personalized medicine in the field of HE.

Several studies in cohorts of patients with advanced HE have demonstrated that those variables that are more closely related to the occurrence of HE are an increase in serum creatinine and a decrease in plasma sodium [38-40]. The most plausible explanation is a decrease in the capacity of the kidney to remove ammonia and an increase in the susceptibility for the developing brain edema, a key component of HE. This finding has lead to propose therapies for circulatory dysfunction to prevent the recurrence of HE.

Patients with procedural portosystemic shunts typically stop suffering gastrointestinal bleeding, but develop HE. Approximately, one third of patients submitted to a TIPS will experience HE [41]. Non-selective portal-systemic shunts (porto-caval, mesocaval) produce more encephalopathy than do selective shunts (distal splenorenal). However, selectivity of splenorenal shunts is lost in the long-term. Elderly patients and those that have poor liver function are at higher risk for post-shunt encephalopathy. There is no hepatic functional test that identifies with confidence those individuals that will develop HE. Reduction of the diameter of TIPS is associated with improvement of HE. Interestingly, patients with loss of portal perfusion before TIPS are protected against post-TIPS HE [42]. 


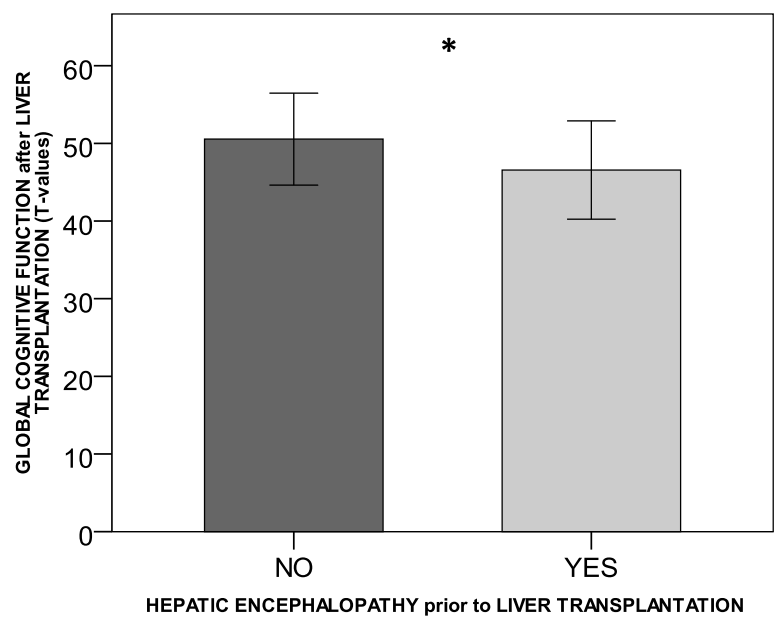

Fig. 3. The development of hepatic encephalopathy before liver transplantation is associated with worse cognitive function after liver transplant (modified from Garcia-Martinez et al. [51]) $\left({ }^{*} p<0.05\right)$.

\section{Sequels of $\mathrm{HE}$}

The metabolic nature of $\mathrm{HE}$ described in the 1950s [43] together with the observation of recovery between episodes led to the traditionally belief that $\mathrm{HE}$ is fully reversible. Recent studies, most of them performed in transplanted patients but also in cohorts of cirrhotic subjects, showed increasing evidence that challenges this classical view.

In a recent study that included a large cohort of cirrhotic patients [44], the authors found that those subjects that developed episodes of HE did not improve psychometric parameters with repeated testing (lack of "learning effect"). The same feature was observed with different psychometric tests in different populations $[40,45]$ confirming persistence of deficits after HE.

Since the implementation of liver transplantation (LT) it has been demonstrated its ability to improves $\mathrm{HE}$, even in patients with severe manifestations [46,47]. However, studies that have assessed neuropsychological function following LT found a heterogeneous outcome with persistent cognitive deficits [48-51]. Many other factors can impact in the postransplant cognitive function such as pretransplant (alcohol aetiology, prior cerebrovascular disease), peritransplant (ischemia) and postransplant events (immunosuppression, infections, stroke... ). One study that performed a prospective assessment up to nine years after LT observed that cognitive function at long-term was associated with vascular risk factors and signs of small-vessel cerebral disease in MR images [52]. However, these recent studies sup- port the notion that $\mathrm{HE}$ is associated with permanent sequels [51].

The origin and nature of these persistence deficits are not well known. Different neuroimaging techniques have shown some degree of brain atrophy in patients with chronic HE $[53,54]$ as well as neuropathological studies [55]. The prevalence and the degree of atrophy were higher among alcoholic patients. This feature could be explained by the fact that alcohol cause a doserelated brain shrink which is partially reversible with abstinence [56]. A recent prospective study performed in a group of patients before and after LT showed an association between prior HE with posttransplant cognitive deficits (Fig. 3) and smaller brain volume. In addition, the smaller brain volume after LT correlated with lower levels of $\mathrm{N}$-acetyl-aspartate/cr considered a neuronal marker [51]. A plausible explanation for these finding is that the chronic exposition to toxins involved in the pathogenesis of HE could cause loss of brain tissue. This hypothesis is supported by the neuropathological demonstration of neuronal loss in the most severe cases of HE [57,58].

The concept that the episodes of HE may lead to irreversible decline in cognitive function has important consequences. It has been recently shown that lactulose and rifaximin decrease the risk of the recurrence of HE [27,32]. Thus, secondary prophylaxis with these drugs is recommended to decrease the number of further episodes of HE. Additional benefits may include preventing neuropsychological decline and may extent to the post-liver transplant period. In addition to this, is important to prevent "premature aging" of the brain by identifying and treating vascular risk factors, such 
as diabetes mellitus and arterial hypertension, and prescribing the minimal possible dose of immunosupressors.

\section{Financial support}

CIBEREHD is supported by Instituto de Salud Carlos III, Madrid, Spain. The data that have generated part of the article have been obtained thanks to the support of FIS 07/0641. Rita García-Martínez has been supported by grant CM07/00109.

\section{References}

[1] J. Cordoba and B. Minguez, Hepatic encephalopathy, Semin Liver Dis 28(1) (2008), 70-80.

[2] P. Ferenci, A. Lockwood, K. Mullen, R. Tarter, K. Weissenborn and A.T. Blei, Hepatic encephalopathy-definition, nomenclature, diagnosis, and quantification: final report of the working party at the 11th World Congresses of Gastroenterology, Vienna, 1998, Hepatology 35(3) (2002), 716-721.

[3] J. Polson and W.M. Lee, AASLD position paper: the management of acute liver failure, Hepatology 41(5) (2005), 1179_ 1197.

[4] G. Ostapowicz, R.J. Fontana, F.V. Schiodt, A. Larson, T.J. Davern, S.H. Han et al., Results of a prospective study of acute liver failure at 17 tertiary care centers in the United States, Ann Intern Med 137(12) (2002), 947-954.

[5] P. Ichai and D. Samuel, Etiology and prognosis of fulminant hepatitis in adults, Liver Transpl 14(Suppl 2) (2008), S67-S79.

[6] D. Samuel and P. Ichai, Prognosis indicator in acute liver failure: Is there a place for cell death markers? J Hepatol 53(4) (2010). 593-595.

[7] J.G. O'Grady, G.J. Alexander, K.M. Hayllar and R. Williams, Early indicators of prognosis in fulminant hepatic failure, Gastroenterology 97(2) (1989), 439-445.

[8] J. Bernuau, A. Goudeau, T. Poynard, F. Dubois, G. Lesage, B. Yvonnet et al., Multivariate analysis of prognostic factors in fulminant hepatitis B, Hepatology 6(4) (1986), 648-651.

[9] A.O. Shakil, D. Kramer, G.V. Mazariegos, J.J. Fung and J. Rakela, Acute liver failure: clinical features, outcome analysis, and applicability of prognostic criteria, Liver Transpl 6(2) (2000), 163-169.

[10] A.C. Anand, P. Nightingale and J.M. Neuberger, Early indicators of prognosis in fulminant hepatic failure: an assessment of the King's criteria, J Hepatol 26(1) (1997), 62-68.

[11] S. Izumi, P.G. Langley, J. Wendon, A.J. Ellis, R.B. Pernambuco, R.D. Hughes et al., Coagulation factor V levels as a prognostic indicator in fulminant hepatic failure, Hepatology 23(6) (1996), 1507-1511.

[12] A. Castells, J.M. Salmeron, M. Navasa, A. Rimola, J. Salo, H. Andreu et al., Liver transplantation for acute liver failure: analysis of applicability, Gastroenterology 105(2) (1993), 532-538.

[13] J.A. Wendon and F.S. Larsen, Intracranial pressure monitoring in acute liver failure. A procedure with clear indications, Hepatology 44(2) (2006), 504-506.
[14] J.M. Llovet, C. Bru and J. Bruix, Prognosis of hepatocellular carcinoma: the BCLC staging classification, Semin Liver Dis 19(3) (1999), 329-338.

[15] G. D'Amico, G. Garcia-Tsao and L. Pagliaro, Natural history and prognostic indicators of survival in cirrhosis: a systematic review of 118 studies, J Hepatol 44(1) (2006), 217-231.

[16] V. Arvaniti, G. D’Amico, G. Fede, P. Manousou, E. Tsochatzis, M. Pleguezuelo et al., Infections in patients with cirrhosis increase mortality four-fold and should be used in determining prognosis, Gastroenterology 139(4) (2010), 12461256, 1256.

[17] R. Jalan and R. Williams, Acute-on-chronic liver failure: pathophysiological basis of therapeutic options, Blood Purif 20(3) (2002), 252-261.

[18] D. Thabut, J. Massard, A. Gangloff, N. Carbonell, C. Francoz, E. Nguyen-Khac et al., Model for end-stage liver disease score and systemic inflammatory response are major prognostic factors in patients with cirrhosis and acute functional renal failure, Hepatology 46(6) (2007), 1872-1882.

[19] M. Cazzaniga, E. Dionigi, G. Gobbo, A. Fioretti, V. Monti and F. Salerno, The systemic inflammatory response syndrome in cirrhotic patients: relationship with their in-hospital outcome, J Hepatol 51(3) (2009), 475-482.

[20] M. Wehler, J. Kokoska, U. Reulbach, E.G. Hahn and R. Strauss, Short-term prognosis in critically ill patients with cirrhosis assessed by prognostic scoring systems, Hepatology 34(2) (2001), 255-261.

[21] T.I. Hassanein, F. Tofteng, R.S. Brown, Jr., B. McGuire, P. Lynch, R. Mehta et al., Randomized controlled study of extracorporeal albumin dialysis for hepatic encephalopathy in advanced cirrhosis, Hepatology 46(6) (2007), 1853-1862.

[22] J. Bustamante, A. Rimola, P.J. Ventura, M. Navasa, I. Cirera, V. Reggiardo et al., Prognostic significance of hepatic encephalopathy in patients with cirrhosis, J Hepatol 30(5) (1999), 890-895.

[23] E. Christensen, J.J. Krintel, S.M. Hansen, J.K. Johansen and E. Juhl, Prognosis after the first episode of gastrointestinal bleeding or coma in cirrhosis. Survival and prognostic factors, Scand J Gastroenterol 24(8) (1989), 999-1006.

[24] J.B. Saunders, J.R. Walters, A.P. Davies and A. Paton, A 20year prospective study of cirrhosis, $\mathrm{Br}$ Med J (Clin Res Ed) 282(6260) (1981), 263-266.

[25] E. Strauss, R. Tramote, E.P. Silva, W.R. Caly, N.Z. Honain, R.A. Maffei et al., Double-blind randomized clinical trial comparing neomycin and placebo in the treatment of exogenous hepatic encephalopathy, Hepatogastroenterology 39(6) (1992). 542-545.

[26] P. Blanc, J.P. Daures, J. Liautard, R. Buttigieg, D. Desprez, G. Pageaux et al., Lactulose-neomycin combination versus placebo in the treatment of acute hepatic encephalopathy. Results of a randomized controlled trial, Gastroenterol Clin Biol 18(12) (1994), 1063-1068.

[27] N.M. Bass, K.D. Mullen, A. Sanyal, F. Poordad, G. Neff, C.B. Leevy et al., Rifaximin treatment in hepatic encephalopathy, N Engl J Med 362(12) (2010), 1071-1081.

[28] C.A. Stewart, M. Malinchoc, W.R. Kim and P.S. Kamath, Hepatic encephalopathy as a predictor of survival in patients with end-stage liver disease, Liver Transpl 13(10) (2007), 13661371.

[29] M. Malinchoc, P.S. Kamath, F.D. Gordon, C.J. Peine, J. Rank and P.C. ter Borg, A model to predict poor survival in patients undergoing transjugular intrahepatic portosystemic shunts, Hepatology 31(4) (2000), 864-871. 
[30] J. Cordoba, G. Olive, J. Alonso, A. Rovira, A. Segarra, M. Perez et al., Improvement of magnetic resonance spectroscopic abnormalities but not pallidal hyperintensity followed amelioration of hepatic encephalopathy after occlusion of a large spleno-renal shunt, J Hepatol 34(1) (2001), 176-178.

[31] O. Riggio, C. Efrati, C. Catalano, F. Pediconi, O. Mecarelli, N. Accornero et al., High prevalence of spontaneous portalsystemic shunts in persistent hepatic encephalopathy: a casecontrol study, Hepatology 42(5) (2005), 1158-1165.

[32] B.C. Sharma, P. Sharma, A. Agrawal and S.K. Sarin, Secondary prophylaxis of hepatic encephalopathy: an open-label randomized controlled trial of lactulose versus placebo, Gastroenterology 137(3) (2009), 885-891.

[33] I.J. Hartmann, M. Groeneweg, J.C. Quero, S.J. Beijeman, R.A. de Man, W.C. Hop et al., The prognostic significance of subclinical hepatic encephalopathy, Am J Gastroenterol 95(8) (2000), 2029-2034.

[34] P. Amodio, F. Del Piccolo, E. Petteno, D. Mapelli, P. Angeli, R. Iemmolo et al., Prevalence and prognostic value of quantified electroencephalogram (EEG) alterations in cirrhotic patients, J Hepatol 35(1) (2001), 37-45.

[35] M. Romero-Gomez, J. Cordoba, R. Jover, J.A. Del Olmo, M. Ramirez, R. Rey et al., Value of the critical flicker frequency in patients with minimal hepatic encephalopathy, Hepatology 45(4) (2007), 879-885.

[36] M. Romero-Gomez, L. Grande and I. Camacho, Prognostic value of altered oral glutamine challenge in patients with minimal hepatic encephalopathy, Hepatology 39(4) (2004), 939943.

[37] M. Romero-Gomez, M. Jover, J.A. Del Campo, J.L. Royo, E. Hoyas, J.J. Galan et al., Variations in the promoter region of the glutaminase gene and the development of hepatic encephalopathy in patients with cirrhosis: a cohort study, Ann Intern Med 153(5) (2010), 281-288.

[38] M. Guevara, M.E. Baccaro, J. Rios, M. Martin-Llahi, J. Uriz, D.A. Ruiz et al., Risk factors for hepatic encephalopathy in patients with cirrhosis and refractory ascites: relevance of serum sodium concentration, Liver Int 30(8) (2010), 11371142 .

[39] O. Riggio, S. Angeloni, F.M. Salvatori, A. De Santis, F. Cerini, A. Farcomeni et al., Incidence, natural history, and risk factors of hepatic encephalopathy after transjugular intrahepatic portosystemic shunt with polytetrafluoroethylene-covered stent grafts, Am J Gastroenterol 103(11) (2008), 2738-2746.

[40] I. Les, E. Doval, R. Garcia-Martinez, M. Planas, G. Cardenas, P. Gomez et al., Effects of branched-chain amino acids supplementation in patients with cirrhosis and a previous episode of hepatic encephalopathy, A randomized study, Am J Gastroenterol (2010), (in press).

[41] G. Pomier-Layrargues, TIPS and hepatic encephalopathy, Semin Liver Dis 16(3) (1996), 315-320.

[42] Z. Hassoun, M. Deschenes, M. Lafortune, M.P. Dufresne, P. Perreault, L. Lepanto et al., Relationship between pre-TIPS liver perfusion by the portal vein and the incidence of postTIPS chronic hepatic encephalopathy, Am J Gastroenterol 96(4) (2001), 1205-1209.

[43] G.B. Phillips, R. Schwartz, G.J. Gabuzda Jr. and C.S. Davidson, The syndrome of impending hepatic coma in patients with cirrhosis of the liver given certain nitrogenous substances, $N$
Engl J Med 247(7) (1952), 239-246.

[44] J.S. Bajaj, C.M. Schubert, D.M. Heuman, J.B. Wade, D.P. Gibson, A. Topaz et al., Persistence of cognitive impairment after resolution of overt hepatic encephalopathy, Gastroenterology 138(7) (2010), 2332-2340.

[45] O. Riggio, L. Ridola, C. Pasquale, S. Nardelli, I. Pentassuglio, F. Moscucci et al., Evidence of Persistent Cognitive Impairment After Resolution of Overt Hepatic Encephalopathy, Clin Gastroenterol Hepatol (2010).

[46] K. Weissenborn, U.J. Tietge, M. Bokemeyer, B. Mohammadi, U. Bode, M.P. Manns et al., Liver transplantation improves hepatic myelopathy: evidence by three cases, Gastroenterology 124(2) (2003), 346-351.

[47] F.S. Larsen, L. Ranek, B.A. Hansen and P. Kirkegaard, Chronic portosystemic hepatic encephalopathy refractory to medical treatment successfully reversed by liver transplantation, Transplant International 8(3) (1995), 246-247.

[48] R.E. Tarter, J.A. Switala, A. Arria, J. Plail and D.H. Van Thiel, Subclinical hepatic encephalopathy. Comparison before and after orthotopic liver transplantation, Transplantation 50(4) (1990), 632-637.

[49] S. Mechtcheriakov, I.W. Graziadei, M. Mattedi, T. Bodner, A. Kugener, H.H. Hinterhubert et al., Incomplete improvement of visuo-motor deficits in patients with minimal hepatic encephalopathy after liver transplantation, Liver Transplantation 10 (2004), 77-83.

[50] F.U. Sotil, J. Gottstein, E. Ayala, C. Randolph and A.T. Blei, Impact of preoperative overt hepatic encephalopathy on neurocognitive function after liver transplantation, Liver Transpl 15(2) (2009), 184-192.

[51] R. Garcia-Martinez, A. Rovira, J. Alonso, C. Jacas, M. SimonTalero, L. Chavarria et al., Hepatic Encephalopathy Is Associated With Posttransplant Cognitive Function and Brain Volume. Liver Transpl 2010; (in press).

[52] M.R. Garcia, A. Rovira, J. Alonso, F.X. Aymerich, E. Huerga, C. Jacas et al., A long-term study of changes in the volume of brain ventricles and white matter lesions after successful liver transplantation, Transplantation 89(5) (2010), 589-594.

[53] M.L. Zeneroli, G. Cioni, C. Vezzelli, S. Grandi, G. Crisi, R. Luzietti et al., Prevalence of brain atrophy in liver cirrhosis patients with chronic persistent encephalopathy. Evaluation by computed tomography, J Hepatol 4(3) (1987), 283-292.

[54] L.L. Levy, Brain atrophy by computerized tomography: does brain matter matter? Hepatology 8(3) (1988), 695-696.

[55] C. Harper and J. Kril, Brain atrophy in chronic alcoholic patients: a quantitative pathological study, J Neurol Neurosurg Psychiatry 48(3) (1985), 211-217.

[56] A. Pfefferbaum, E.V. Sullivan, D.H. Mathalon, P.K. Shear, M.J. Rosenbloom and K.O. Lim, Longitudinal changes in magnetic resonance imaging brain volumes in abstinent and relapsed alcoholics, Alcohol Clin Exp Res 19(5) (1995), 11771191.

[57] M. Victor, R.D. ADAMS and M. Cole, The acquired (nonWilsonian) type of chronic hepatocerebral degeneration, Medicine (Baltimore) 44(5) (1965), 345-396.

[58] M.H. Finlayson and B. Superville, Distribution of cerebral lesions in acquired hepatocerebral degeneration, Brain 104 $(\mathrm{Pt}$ 1) (1981), 79-95. 


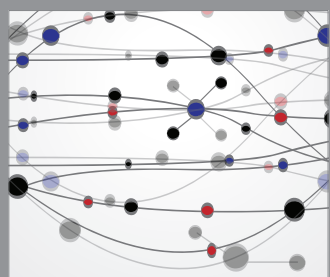

The Scientific World Journal
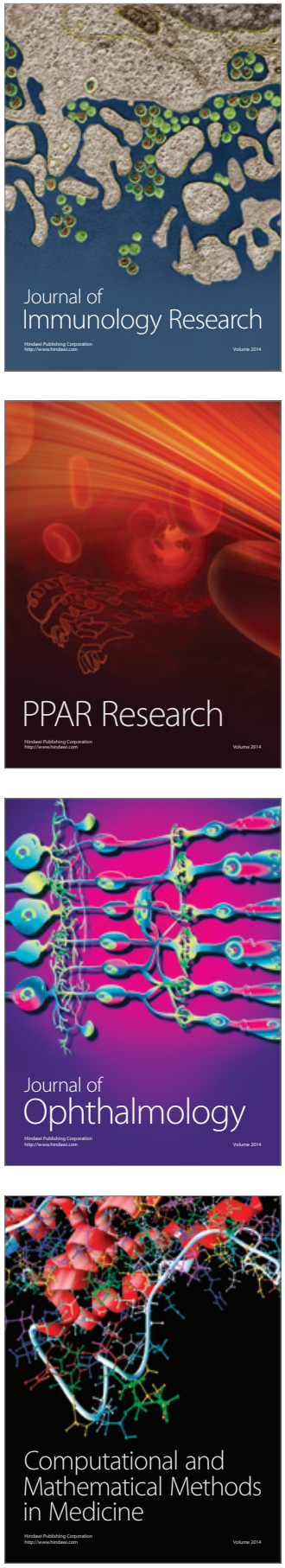

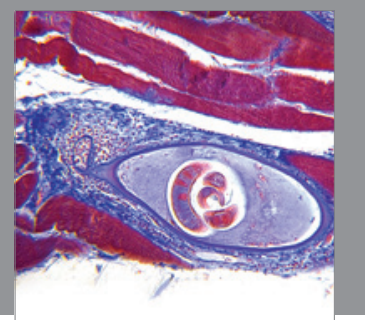

Gastroenterology

Research and Practice
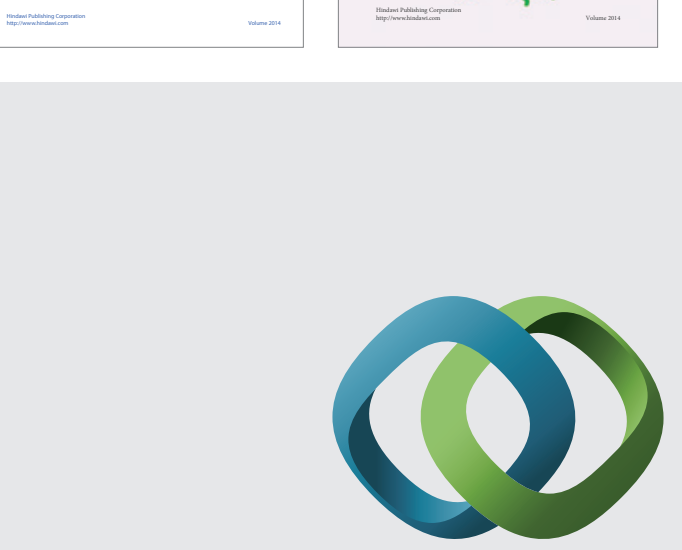

\section{Hindawi}

Submit your manuscripts at

http://www.hindawi.com
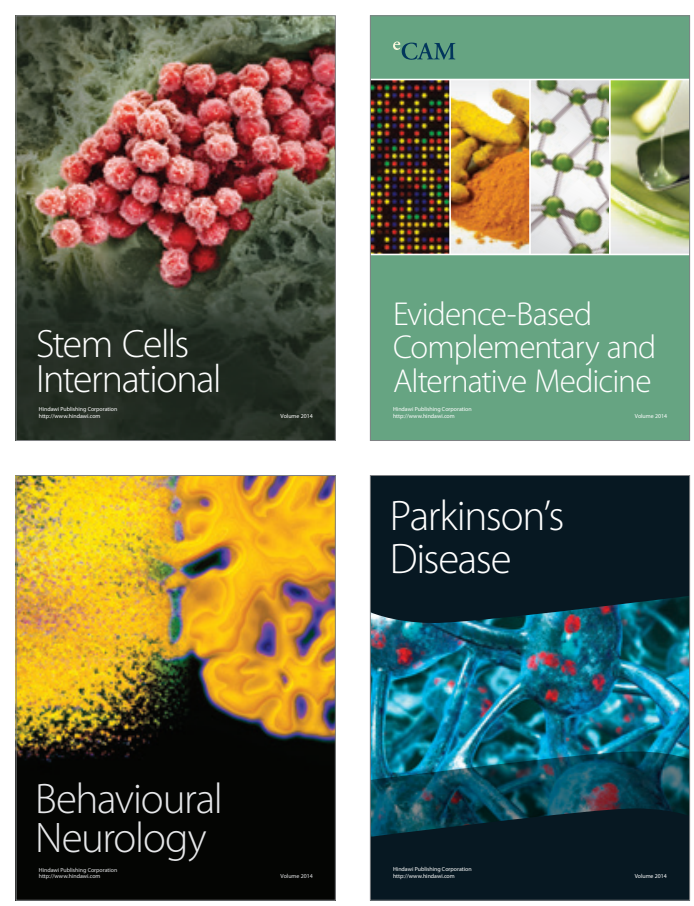

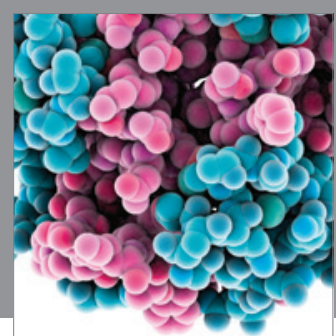

Journal of
Diabetes Research

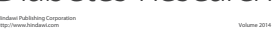

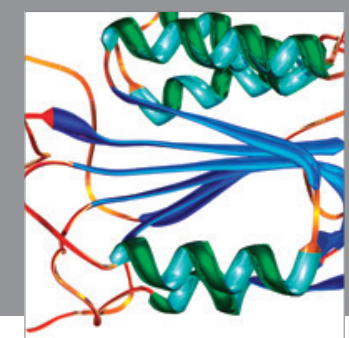

Disease Markers
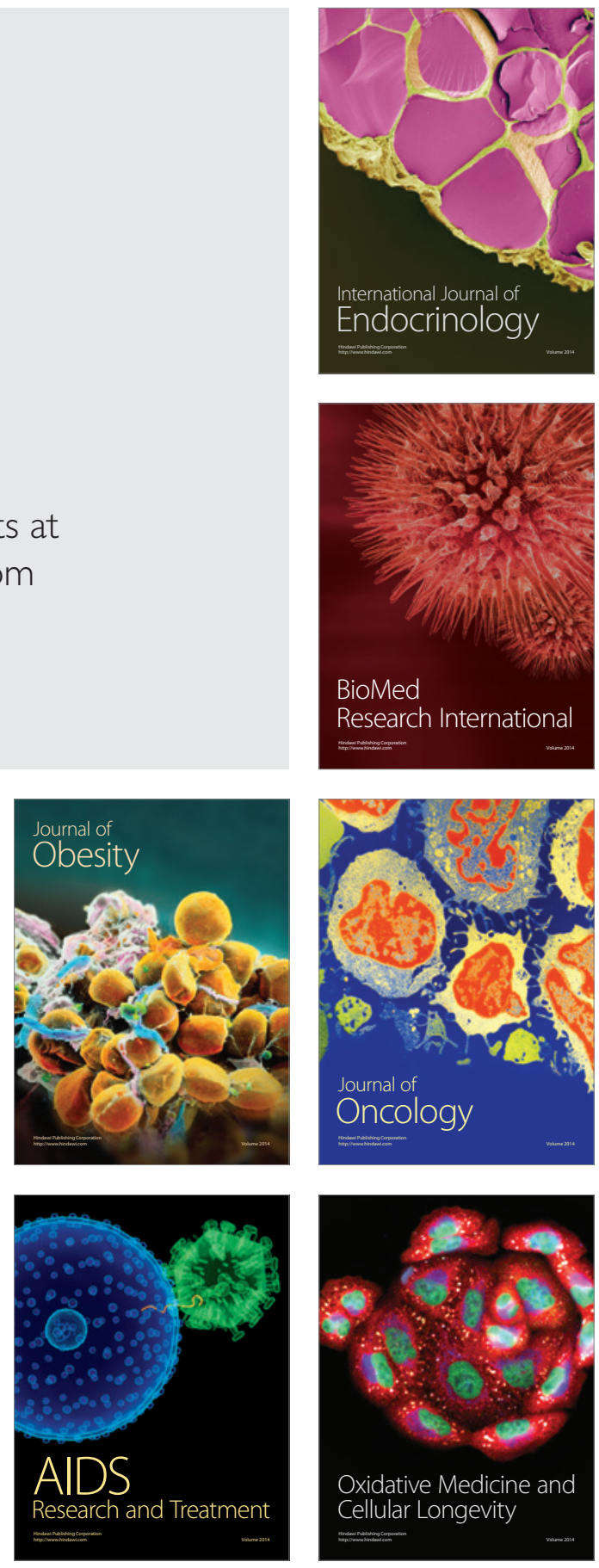